\title{
Formalization of Integral Linear Space ${ }^{1}$
}

\author{
Yuichi Futa \\ Shinshu University \\ Nagano, Japan
}

\author{
Hiroyuki Okazaki \\ Shinshu University \\ Nagano, Japan
}

\author{
Yasunari Shidama \\ Shinshu University \\ Nagano, Japan
}

Summary. In this article, we formalize integral linear spaces, that is a linear space with integer coefficients. Integral linear spaces are necessary for lattice problems, LLL (Lenstra-Lenstra-Lovász) base reduction algorithm that outputs short lattice base and cryptographic systems with lattice [8].

MML identifier: RLVECT_X, version: $\underline{7.11 .07 \quad 4.156 .1112}$

The notation and terminology used here have been introduced in the following papers: [1], [10], [3], [9], [11], [2], [4], [6], [16], [14], [13], [12], [5], [7], [15], and $[17]$.

\section{Preliminaries}

The following propositions are true:

(1) Let $X$ be a real linear space and $R_{1}, R_{2}$ be finite sequences of elements of $X$. If len $R_{1}=$ len $R_{2}$, then $\sum\left(R_{1}+R_{2}\right)=\sum R_{1}+\sum R_{2}$.

(2) Let $X$ be a real linear space and $R_{1}, R_{2}, R_{3}$ be finite sequences of elements of $X$. If len $R_{1}=$ len $R_{2}$ and $R_{3}=R_{1}-R_{2}$, then $\sum R_{3}=\sum R_{1}-$ $\sum R_{2}$.

(3) Let $X$ be a real linear space, $R_{1}, R_{2}$ be finite sequences of elements of $X$, and $a$ be an element of $\mathbb{R}$. If $R_{2}=a R_{1}$, then $\sum R_{2}=a \cdot \sum R_{1}$.

\footnotetext{
${ }^{1}$ This work was supported by JSPS KAKENHI 22300285.
} 


\section{Integral Linear Space}

For simplicity, we use the following convention: $x$ denotes a set, $a$ denotes a real number, $i$ denotes an integer, $V$ denotes a real linear space, $v, v_{1}, v_{2}, v_{3}$, $u, w, w_{1}, w_{2}, w_{3}$ denote vectors of $V, A, B$ denote subsets of $V, L$ denotes a linear combination of $V$, and $l, l_{1}, l_{2}$ denote linear combinations of $A$.

Let us consider $V, i, L$. The functor $i \cdot L$ yielding a linear combination of $V$ is defined as follows:

(Def. 1) For every $v$ holds $(i \cdot L)(v)=i \cdot L(v)$.

Let us consider $V, A$. The functor $\operatorname{Lin}_{\mathbb{Z}} A$ yielding a subset of $V$ is defined by:

(Def. 2) $\operatorname{Lin}_{\mathbb{Z}} A=\left\{\sum l: \operatorname{rng} l \subseteq \mathbb{Z}\right\}$.

One can prove the following propositions:

(4) $(i) \cdot l=i \cdot l$.

(5) If $\operatorname{rng} l_{1} \subseteq \mathbb{Z}$ and $\operatorname{rng} l_{2} \subseteq \mathbb{Z}$, then $\operatorname{rng}\left(l_{1}+l_{2}\right) \subseteq \mathbb{Z}$.

(6) If $\operatorname{rng} l \subseteq \mathbb{Z}$, then $\operatorname{rng}(i \cdot l) \subseteq \mathbb{Z}$.

(7) $\operatorname{rng}\left(\mathbf{0}_{\mathrm{LC}_{V}}\right) \subseteq \mathbb{Z}$.

(8) $\operatorname{Lin}_{\mathbb{Z}} A \subseteq$ the carrier of $\operatorname{Lin}(A)$.

(9) If $v, u \in \operatorname{Lin}_{\mathbb{Z}} A$, then $v+u \in \operatorname{Lin}_{\mathbb{Z}} A$.

(10) If $v \in \operatorname{Lin}_{\mathbb{Z}} A$, then $i \cdot v \in \operatorname{Lin}_{\mathbb{Z}} A$.

(11) $0_{V} \in \operatorname{Lin}_{\mathbb{Z}} A$.

(12) If $x \in A$, then $x \in \operatorname{Lin}_{\mathbb{Z}} A$.

(13) If $A \subseteq B$, then $\operatorname{Lin}_{\mathbb{Z}} A \subseteq \operatorname{Lin}_{\mathbb{Z}} B$.

(14) $\operatorname{Lin}_{\mathbb{Z}}(A \cup B)=\left(\operatorname{Lin}_{\mathbb{Z}} A\right)+\operatorname{Lin}_{\mathbb{Z}} B$.

(15) $\operatorname{Lin}_{\mathbb{Z}}(A \cap B) \subseteq\left(\operatorname{Lin}_{\mathbb{Z}} A\right) \cap \operatorname{Lin}_{\mathbb{Z}} B$.

(16) $x \in \operatorname{Lin}_{\mathbb{Z}}\{v\}$ iff there exists an integer $a$ such that $x=a \cdot v$.

(17) $v \in \operatorname{Lin}_{\mathbb{Z}}\{v\}$.

(18) $x \in v+\operatorname{Lin}_{\mathbb{Z}}\{w\}$ iff there exists an integer $a$ such that $x=v+a \cdot w$.

(19) $x \in \operatorname{Lin}_{\mathbb{Z}}\left\{w_{1}, w_{2}\right\}$ iff there exist integers $a, b$ such that $x=a \cdot w_{1}+b \cdot w_{2}$.

(20) $w_{1} \in \operatorname{Lin}_{\mathbb{Z}}\left\{w_{1}, w_{2}\right\}$.

(21) $x \in v+\operatorname{Lin}_{\mathbb{Z}}\left\{w_{1}, w_{2}\right\}$ iff there exist integers $a, b$ such that $x=v+a$. $w_{1}+b \cdot w_{2}$.

(22) $x \in \operatorname{Lin}_{\mathbb{Z}}\left\{v_{1}, v_{2}, v_{3}\right\}$ iff there exist integers $a, b, c$ such that $x=a \cdot v_{1}+$ $b \cdot v_{2}+c \cdot v_{3}$.

(23) $w_{1}, w_{2}, w_{3} \in \operatorname{Lin}_{\mathbb{Z}}\left\{w_{1}, w_{2}, w_{3}\right\}$.

(24) $x \in v+\operatorname{Lin}_{\mathbb{Z}}\left\{w_{1}, w_{2}, w_{3}\right\}$ iff there exist integers $a, b, c$ such that $x=$ $v+a \cdot w_{1}+b \cdot w_{2}+c \cdot w_{3}$. 
(25) Let $x$ be a set. Then $x \in \operatorname{Lin}_{\mathbb{Z}} A$ if and only if there exist finite sequences $g_{1}, h_{1}$ of elements of $V$ and there exists an integer-valued finite sequence $a_{1}$ such that $x=\sum h_{1}$ and $\operatorname{rng} g_{1} \subseteq A$ and len $g_{1}=\operatorname{len} h_{1}$ and len $g_{1}=\operatorname{len} a_{1}$ and for every natural number $i$ such that $i \in \operatorname{Seg} \operatorname{len} g_{1}$ holds $\left(h_{1}\right)_{i}=$ $a_{1}(i) \cdot\left(g_{1}\right)_{i}$.

Let $R_{4}$ be a real linear space and let $f$ be a finite sequence of elements of $R_{4}$. The functor $\operatorname{Lin}_{\mathbb{Z}} f$ yielding a subset of $R_{4}$ is defined by the condition (Def. 3).

(Def. 3) $\operatorname{Lin}_{\mathbb{Z}} f=\left\{\sum g ; g\right.$ ranges over len $f$-element finite sequences of elements of $R_{4}: \bigvee_{a}$ : len $f$-element integer-valued finite sequence $\bigwedge_{i}$ :natural number $(i \in$ Seg len $\left.\left.f \Rightarrow g_{i}=a(i) \cdot f_{i}\right)\right\}$.

One can prove the following propositions:

(26) Let $R_{4}$ be a real linear space, $f$ be a finite sequence of elements of $R_{4}$, and $x$ be a set. Then $x \in \operatorname{Lin}_{\mathbb{Z}} f$ if and only if there exists a len $f$-element finite sequence $g$ of elements of $R_{4}$ and there exists a len $f$-element integervalued finite sequence $a$ such that $x=\sum g$ and for every natural number $i$ such that $i \in$ Seg len $f$ holds $g_{i}=a(i) \cdot f_{i}$.

(27) Let $R_{4}$ be a real linear space, $f$ be a finite sequence of elements of $R_{4}$, $x, y$ be elements of $R_{4}$, and $a, b$ be elements of $\mathbb{Z}$. If $x, y \in \operatorname{Lin}_{\mathbb{Z}} f$, then $a \cdot x+b \cdot y \in \operatorname{Lin}_{\mathbb{Z}} f$.

(28) For every real linear space $R_{4}$ and for every finite sequence $f$ of elements of $R_{4}$ such that $f=$ Seg len $f \longmapsto 0_{\left(R_{4}\right)}$ holds $\sum f=0_{\left(R_{4}\right)}$.

(29) Let $R_{4}$ be a real linear space, $f$ be a finite sequence of elements of $R_{4}$, $v$ be an element of $R_{4}$, and $i$ be a natural number. If $i \in \operatorname{Seg}$ len $f$ and $f=\left(\right.$ Seg len $\left.f \longmapsto 0_{\left(R_{4}\right)}\right)+\cdot(\{i\} \longmapsto v)$, then $\sum f=v$.

(30) Let $R_{4}$ be a real linear space, $f$ be a finite sequence of elements of $R_{4}$, and $i$ be a natural number. If $i \in \operatorname{Seg} \operatorname{len} f$, then $f_{i} \in \operatorname{Lin}_{\mathbb{Z}} f$.

(31) For every real linear space $R_{4}$ and for every finite sequence $f$ of elements of $R_{4}$ holds $\operatorname{rng} f \subseteq \operatorname{Lin}_{\mathbb{Z}} f$.

(32) Let $R_{4}$ be a real linear space, $f$ be a non empty finite sequence of elements of $R_{4}, g, h$ be finite sequences of elements of $R_{4}$, and $s$ be an integer-valued finite sequence. Suppose $\operatorname{rng} g \subseteq \operatorname{Lin}_{\mathbb{Z}} f$ and len $g=\operatorname{len} s$ and len $g=\operatorname{len} h$ and for every natural number $i$ such that $i \in \operatorname{Seg}$ len $g$ holds $h_{i}=s(i) \cdot g_{i}$. Then $\sum h \in \operatorname{Lin}_{\mathbb{Z}} f$.

(33) For every real linear space $R_{4}$ and for every non empty finite sequence $f$ of elements of $R_{4}$ holds $\operatorname{Lin}_{\mathbb{Z}} \operatorname{rng} f=\operatorname{Lin}_{\mathbb{Z}} f$.

(34) $\quad \operatorname{Lin}\left(\operatorname{Lin}_{\mathbb{Z}} A\right)=\operatorname{Lin}(A)$.

(35) Let $x$ be a set, $g_{1}, h_{1}$ be finite sequences of elements of $V$, and $a_{1}$ be an integer-valued finite sequence. Suppose $x=\sum h_{1}$ and $\operatorname{rng} g_{1} \subseteq \operatorname{Lin}_{\mathbb{Z}} A$ and len $g_{1}=\operatorname{len} h_{1}$ and len $g_{1}=$ len $a_{1}$ and for every natural number $i$ such that $i \in \operatorname{Seg}$ len $g_{1}$ holds $\left(h_{1}\right)_{i}=a_{1}(i) \cdot\left(g_{1}\right)_{i}$. Then $x \in \operatorname{Lin}_{\mathbb{Z}} A$. 
(36) $\operatorname{Lin}_{\mathbb{Z}} \operatorname{Lin}_{\mathbb{Z}} A=\operatorname{Lin}_{\mathbb{Z}} A$.

(37) If $\operatorname{Lin}_{\mathbb{Z}} A=\operatorname{Lin}_{\mathbb{Z}} B$, then $\operatorname{Lin}(A)=\operatorname{Lin}(B)$.

\section{REFERENCES}

[1] Grzegorz Bancerek. Cardinal numbers. Formalized Mathematics, 1(2):377-382, 1990.

[2] Grzegorz Bancerek. The fundamental properties of natural numbers. Formalized Mathematics, 1(1):41-46, 1990.

[3] Grzegorz Bancerek and Krzysztof Hryniewiecki. Segments of natural numbers and finite sequences. Formalized Mathematics, 1(1):107-114, 1990.

[4] Czesław Byliński. Partial functions. Formalized Mathematics, 1(2):357-367, 1990.

[5] Noboru Endou, Takashi Mitsuishi, and Yasunari Shidama. Dimension of real unitary space. Formalized Mathematics, 11(1):23-28, 2003.

[6] Krzysztof Hryniewiecki. Basic properties of real numbers. Formalized Mathematics, 1(1):35-40, 1990.

[7] Jarosław Kotowicz. Real sequences and basic operations on them. Formalized Mathematics, 1(2):269-272, 1990.

[8] Daniele Micciancio and Shafi Goldwasser. Complexity of lattice problems: A cryptographic perspective (the international series in engineering and computer science). 2002.

[9] Andrzej Trybulec. Binary operations applied to functions. Formalized Mathematics, 1(2):329-334, 1990.

[10] Andrzej Trybulec. Domains and their Cartesian products. Formalized Mathematics, 1(1):115-122, 1990.

[11] Michał J. Trybulec. Integers. Formalized Mathematics, 1(3):501-505, 1990.

[12] Wojciech A. Trybulec. Basis of real linear space. Formalized Mathematics, 1(5):847-850, 1990 .

[13] Wojciech A. Trybulec. Linear combinations in real linear space. Formalized Mathematics, 1(3):581-588, 1990.

[14] Wojciech A. Trybulec. Vectors in real linear space. Formalized Mathematics, 1(2):291-296, 1990.

[15] Zinaida Trybulec. Properties of subsets. Formalized Mathematics, 1(1):67-71, 1990.

[16] Edmund Woronowicz. Relations defined on sets. Formalized Mathematics, 1(1):181-186, 1990.

[17] Hiroshi Yamazaki and Yasunari Shidama. Algebra of vector functions. Formalized Mathematics, 3(2):171-175, 1992.

Received August 17, 2010 